\title{
On the anisotropy of the turbulent passive scalar in the presence of a mean scalar gradient
}

\author{
Wouter J.T. Bos \\ LMFA-CNRS, Université de Lyon, Ecole Centrale de Lyon, Ecully, \\ France
}

$31 / 08 / 2013$

\begin{abstract}
We investigate the origin of the scalar gradient skewness in isotropic turbulence on which a mean scalar gradient is imposed. The problem of the advection of an anisotropic scalar field is reformulated in terms of the advection of an isotropic vector field. For this field triadic closure equations are derived. It is shown how the scaling of the scalar gradient skewness depends on the choice of the timescale used for the Lagrangian decorrelation of the vector field. The persistent anisotropy in the small scales for the third-order statistics is shown to be perfectly compatible with Corrsin-Obukhov scaling for second-order quantities, since secondand third-order scalar quantities are governed by a different triad correlation timescale. Whereas the inertial range dynamics of second-order scalar quantities are governed by the Lagrangian velocity correlation time, the third-order quantities remain correlated over a time related to the largescale dynamics of the scalar field. It is argued that this time is determined by the average time it takes for a fluid particle to travel between ramp-cliff scalar structures.
\end{abstract}

\section{Introduction}

Statistical isotropy is a prerequisite for a large number of theoretical descriptions of the dynamics of turbulent flows. Purely isotropic flows are however rare in practice. Even in the absence of inhomogeneity, anisotropy, as introduced by linear or nonlinear forcing mechanism such as density gradients, rotation, magnetic fields or imposed velocity gradients, leads to a dynamics which is even harder to capture by statistical theory or models than the isotropic case. The complexity of anisotropic turbulence is mainly rooted in the intricate interplay between the nonlinearity of the Navier-Stokes equations and the anisotropic, linear forcing terms. In some special cases, when the timescale of the linear forcing mechanism is much smaller than the nonlinear timescale, linear approximations 
can be used, such as rapid distortion theory [1], [2], in which nonlinearity is ignored. Another approach is linear response theory, where the anisotropy is treated as a perturbation of the isotropic, nonlinearity dominated state $[3,4]$. However, a general theory of anisotropic turbulence is currently not available, and each particular forcing mechanism leads to a dynamics with its own characteristics (e.g. [5]). In this context, the significance of the theory of isotropic turbulence is due to the fact that in a large number of cases the anisotropy becomes less important when one focuses on small enough scales. This postulate of local isotropy [6] is a corner stone of the phenomenological description of turbulence, because it states that at least the small scales of a wide range of turbulent flows share some universal features.

The dynamics of a passive scalar in a turbulent flow, governed by a linear advection-diffusion equation, bares some similarities with the turbulence problem. The simplest case of statistical anisotropy for the passive scalar is its advection by an isotropic turbulence in the presence of a linear scalar concentration profile which is constant is time. This case, first studied by [7], has proven to be far more difficult than could be expected initially. Indeed, it was observed that the small scales of the passive scalar advected by a turbulent velocity field displayed a persisting anisotropy, as measured by the scalar gradient skewness $[8,9]$, thereby challenging the local isotropy postulate for scalar turbulence [10]. It was shown that this anisotropy was not induced by the anisotropic large scale fluid flow, since it was also observed in nearly isotropic wind tunnel experiments (e.g.[11]) and Direct Numerical Simulations at intermediate Reynolds numbers [12]. Also, the persistent skewness did not seem to depend on the details of the advecting flow since even in a "structureless" synthetic flow (delta-correlated Gaussian noise), the same phenomenon was observed [13]. The situation tends to be different from the small scale anisotropy in shear-flow. In that case a non-zero transverse velocity derivative skewness was observed at low Reynolds numbers [14], which seemed to vanish at large Reynolds numbers [15, 16]. This is not so for the scalar gradient skewness, which does not seem to decrease for high Reynolds (or Péclet) number. Several theoretical approaches have been applied to analyze the origin of this persisting anisotropy. For instance, the skewness was analyzed using the $S O(3)$ method [17], and three-point correlation functions were introduced in [18] and experimentally analyzed in [19] to determine the origin of the scalar skewness. These studies have led to a partial understanding of the persistent small-scale anisotropy of the passive scalar and its relation to the postulate of local isotropy.

In the present work we will contribute to the understanding of the origin of this small scale anisotropy. The novelty of the present work is two-fold. Firstly we reformulate the anisotropic scalar problem as an isotropic vectorial problem. Secondly we derive, using techniques of the Direct Interaction Approximation (DIA) type, a closed expression for the scalar gradient skewness as a function of second-order wavenumber spectra, which allows to show how the non-vanishing scalar-gradient skewness is directly related to the Lagrangian correlation of the scalar fluctuations.

In section 2 we introduce the governing equations and we recall the classical 
scaling of spectra and structure functions within the context of KolmogorovCorrsin-Obukhov phenomenology. Then, in section 3 the kinematics of the structure functions and their relation to wave-vector spectra is discussed, introducing the diffusive displacement field to reformulate the anisotropic scalar problem as an isotropic vectorial problem. Closed expressions are obtained for the different quantities in section 4 and the details of this closure are given in the appendices. The closure-expressions contain timescales which need to be modeled. The influence of the choice of these timescales is discussed in section 5 where it will be argued that the non-vanishing skewness is compatible with Corrsin-Obukhov phenomenology since different timescales govern the dynamics of the second- and third-order scalar structure functions. Results from numerical integration are reported in section 6 and some discussion on the interpretation of the results is presented in section 7 .

\section{Structure functions and scaling}

\subsection{Structure functions}

We will consider a passive scalar $\Theta(\boldsymbol{x}, t)$ advected by an incompressible isotropic velocity field $\boldsymbol{u}(\boldsymbol{x}, t)$,

$$
\partial_{t} \Theta+\boldsymbol{u} \cdot \nabla \Theta=D \Delta \Theta .
$$

In the present study we only consider unity Prandtl number, $\operatorname{Pr}=\nu / D$, so that the kinematic viscosity $\nu$ and the thermal diffusion coefficient $D$ have the same value. Here and in the following, the time- and coordinate-dependence of the different quantities will be omitted from our notation, unless several timeinstants or coordinates appear in the same expression. We consider the case in which the velocity field is turbulent, and the flow is statistically isotropic and incompressible. The passive scalar consists of a mean stationary part, and a fluctuation $\theta$. The mean part is chosen to be given by a uniform scalar gradient $\boldsymbol{\Gamma}=\nabla\langle\Theta\rangle$ and is defined to be zero at $\boldsymbol{x}=0$, so that

$$
\Theta=\boldsymbol{\Gamma} \cdot \boldsymbol{x}+\theta .
$$

The equation for the scalar fluctuation is then,

$$
\partial_{t} \theta+\boldsymbol{u} \cdot \nabla \theta=D \Delta \theta-\boldsymbol{\Gamma} \cdot \boldsymbol{u} .
$$

For convenience we can choose the scalar gradient in the $z$-direction and of value -1 , so that the equation reads,

$$
\partial_{t} \theta+\boldsymbol{u} \cdot \nabla \theta=D \Delta \theta+\boldsymbol{u}_{3}
$$

This does not induce any loss of generality since the scalar equation is linear in $\theta$.

The statistical distribution of the passive scalar $\theta$ over different lengthscales can be quantified by the scalar structure functions, given by

$$
D_{\theta}^{(n)}(\boldsymbol{x}, \boldsymbol{r})=\left\langle\delta_{\theta}(\boldsymbol{x}, \boldsymbol{r})^{n}\right\rangle
$$


with

$$
\delta_{\theta}(\boldsymbol{x}, \boldsymbol{r})=\theta(\boldsymbol{x})-\theta(\boldsymbol{x}+\boldsymbol{r}) .
$$

where $\boldsymbol{r}$ is the separation distance between two points and the angular brackets denote an ensemble average. In a statistically homogeneous scalar field (as is the fluctuation field in our case) the averaged quantities will be independent of $\boldsymbol{x}$, so that

$$
D_{\theta}^{(n)}(\boldsymbol{x}, \boldsymbol{r})=D_{\theta}^{(n)}(\boldsymbol{r})
$$

In the isotropic case the angular dependence also vanishes so that the structure function depends only on the modulus $r$. In addition, in this case all odd order structure functions (such as $D_{\theta}^{(3)}(\boldsymbol{r})$ ) are identically zero due to symmetry. In the anisotropic case, which we consider here, the odd-order structure functions do not necessarily vanish and the structure functions do not only depend on the separation distance $r$, but also on the angle between the direction of the mean gradient and the vector $\boldsymbol{r}$. Let us, to characterize the anisotropy of the scalar field, focus on two directions. First, the direction parallel to the direction of the gradient, second, perpendicular to the gradient. The statistics corresponding to these directions will be indicated by the subscripts $\|$ and $\perp$, respectively,

$$
\begin{gathered}
D_{\theta \|}^{(n)}(r)=\left\langle\delta_{\theta}\left(\boldsymbol{r}_{\|}, t\right)^{n}\right\rangle \\
D_{\theta \perp}^{(n)}(r)=\left\langle\delta_{\theta}\left(\boldsymbol{r}_{\perp}, t\right)^{n}\right\rangle,
\end{gathered}
$$

where $\boldsymbol{r}_{\|}=\left(\boldsymbol{r} \cdot \boldsymbol{e}_{\Gamma}\right) \boldsymbol{e}_{\Gamma}$ and $\boldsymbol{r}_{\perp}=\boldsymbol{r} \times \boldsymbol{e}_{\Gamma}$ and where $\boldsymbol{e}_{\Gamma}$ is the unit vector in the direction of the mean scalar gradient. It follows from axisymmetry that $D_{\theta \perp}^{(3)}(r)$ and all other odd order perpendicular structure functions are zero. The parallel quantity $D_{\theta \|}^{(3)}(r)$, is however not zero by symmetry. Since this quantity is zero in an isotropic scalar field, it can be used to measure the departure from isotropy as a function of scale. In particular its normalized value, the parallel skewness, is often used to measure the return to isotropy in the small scales. This skewness is defined as

$$
S_{\theta}(r)=\frac{D_{\theta \|}^{(3)}(r)}{\left(D_{\theta \|}^{(2)}(r)\right)^{3 / 2}} .
$$

It is this gradient skewness which is persistent at high Reynolds numbers, as discussed in the introduction, which seems to contradict the local isotropy hypothesis.

Similarly, the velocity-field can be characterized by the velocity structure functions,

$$
\begin{gathered}
D_{u \|}^{(n)}(r)=\left\langle\delta_{u}\left(\boldsymbol{r}_{\|}, t\right)^{n}\right\rangle \\
D_{u \perp}^{(n)}(r)=\left\langle\delta_{u}\left(\boldsymbol{r}_{\perp}, t\right)^{n}\right\rangle,
\end{gathered}
$$

where $\delta_{u}(\boldsymbol{r})$ is the increment of one component of the velocity and the directions $\perp$ and $\|$ are now defined with respect to the direction of the considered component of the velocity vector. 


\section{2 wavenumber spectra and self-similar scaling}

In this section we introduce wavenumber spectra, some of which are the Fourierspace analogues of the structure functions discussed in the previous section. The wavenumber distribution of kinetic energy and scalar variance are given, respectively, by the energy and scalar spectra, defined such that

$$
\begin{array}{r}
\int E(k) d k=\frac{1}{2} \overline{u_{i} u_{i}} \\
\int E_{\theta}(k) d k=\overline{\theta^{2}} .
\end{array}
$$

The scalar flux spectrum is equivalently defined such that

$$
\int F_{u \theta}(k) d k=\overline{u_{3} \theta}
$$

If Kolmogorov's similarity hypothesis is satisfied [6], energy spectra should scale in the inertial and dissipation range as

$$
E(k)=\nu^{5 / 4} \epsilon^{1 / 4} \tilde{E}(k \eta)
$$

where $\tilde{E}(k \eta)$ is a dimensionless function independent of the large-scale flow. Plotting $\tilde{E}(k \eta)$ should therefore collapse the results for different flows and Reynolds numbers in the inertial and dissipation range. In the inertial range, where $E(k)$ is independent of the viscosity, dimensional analysis leads to

$$
E(k) \sim \epsilon^{2 / 3} k^{-5 / 3} .
$$

Similarly for the velocity structure-functions we have,

$$
D_{u}^{(n)}(r)=(\nu \epsilon)^{n / 4} \tilde{D}_{u}^{(n)}(r / \eta)
$$

and in the inertial range,

$$
D_{u}^{(n)}(r) \sim(\epsilon r)^{n}
$$

If Corrsin-Obukhov phenomenology holds [20, 10], the scalar spectra and (both parallel and perpendicular) scalar second-order structure functions should behave as

$$
\begin{array}{r}
E_{\theta}(k)=\epsilon_{\theta} \nu^{5 / 4} \epsilon^{3 / 4} \tilde{E}_{\theta}(k \eta) \\
D_{\theta}^{(2)}(r)=\epsilon_{\theta}(\nu \epsilon)^{1 / 2} \tilde{D}_{\theta}^{(2)}(r / \eta),
\end{array}
$$

which gives in the inertial-convective range,

$$
\begin{aligned}
& E_{\theta}(k) \sim \epsilon_{\theta} \epsilon^{-1 / 3} k^{-5 / 3} \\
& D_{\theta}^{(2)}(r) \sim \epsilon_{\theta} \epsilon^{-1 / 3} r^{2 / 3},
\end{aligned}
$$


where we assumed the scaling to be independant of $\nu$ and $D$. The scalar-velocity cross-correlation spectrum is non-zero only if a mean scalar gradient is present. Following [21], its scaling should be proportional to the strength of this gradient, and further determined by $\epsilon$ and $k$,

$$
F_{u \theta}(k)=\Gamma \nu^{7 / 4} \epsilon^{1 / 4} \tilde{F}_{u_{\theta}}(k \eta) ; \quad F_{u \theta}(k) \sim \Gamma \epsilon^{1 / 3} k^{-7 / 3} .
$$

If we assume the third-order structure-function to be proportional to the scalar gradient, it should dimensionally be proportional to the scalar dissipation $\epsilon_{\theta}$, to give,

$$
D_{\theta \|}^{(3)}(r)=\Gamma \epsilon_{\theta} \nu^{5 / 4} \epsilon^{-3 / 4} \tilde{D}_{\theta \|}^{(3)}(r / \eta)
$$

so that, if $D_{\theta \|}^{(3)}(r)$ is to be independent of the viscosity and diffusivity in the inertial range, it should scale as

$$
D_{\theta \|}^{(3)}(r) \sim \Gamma \epsilon_{\theta} \epsilon^{-1 / 3} r^{5 / 3}
$$

so that

$$
S_{\theta}(r) \sim \Gamma \epsilon_{\theta}^{-1 / 2} \epsilon^{1 / 6} r^{2 / 3}
$$

This implies that the at very small scales the scalar gradient skewness should tend to zero. This is not what is observed in experiments and simulations and we will investigate the origin of this anomalous behaviour in the present work.

\section{Kinematics of an advected vector field}

\subsection{Advection of an isotropic displacement vector-field}

The equation for the advection of a passive scalar fluctuation, equation (3), contains a source-term proportional to $\boldsymbol{\Gamma} \cdot \boldsymbol{u}$. This source term for scalar fluctuations is anisotropic, and so will be the scalar field. It is possible to reformulate this anisotropic problem as an isotropic problem. The price to pay is that instead of a scalar field, we will now have to consider an advected vector field. The isotropic vector-field that we introduce is the diffusive displacement field. The dynamics of this field is governed by the equation,

$$
\partial_{t} \boldsymbol{\chi}+\boldsymbol{u} \cdot \nabla \boldsymbol{\chi}=D \Delta \boldsymbol{\chi}+\boldsymbol{u}
$$

The quantity $\chi(\boldsymbol{x}, t)$ can be interpreted physically as the displacement distance of a fluid particle with respect to its initial position, but with a diffusive term which limits the memory with respect to its initial position. A more extensive description of the displacement vector and its relation to Lagrangian dynamics can be found in [22]. By identifying $\chi_{3}=\theta$, we see that (4) describes the evolution of one component of (23). Since (23) contains now an isotropic source term (the last term of equation (23)), the statistics of the vector-field will be isotropic if the initial conditions are. We will consider an initial field with 
zero fluctuations, which is obviously isotropic. The displacement vector-field contains thus all the dynamics of the scalar fluctuations, and is, in the absence of other effects, perfectly statistically isotropic.

The second- and third-order parallel and perpendicular structure functions of the scalar can then be related to the second- and third-order correlation functions,

$$
\begin{array}{r}
C_{i j}(\boldsymbol{r})=\left\langle\chi_{i}(\boldsymbol{x}) \chi_{j}(\boldsymbol{x}+\boldsymbol{r})\right\rangle \\
C_{i j m}(\boldsymbol{r})=\left\langle\chi_{i}(\boldsymbol{x}) \chi_{j}(\boldsymbol{x}) \chi_{m}(\boldsymbol{x}+\boldsymbol{r})\right\rangle,
\end{array}
$$

by considering the longitudinal and transversal components, according to the following relations,

$$
\begin{array}{r}
D_{\theta \|}^{(2)}(r)=2 \frac{r_{i} r_{j}}{r^{2}}\left(C_{i j}(0)-C_{i j}(\boldsymbol{r})\right) \\
D_{\theta \perp}^{(2)}(r)=\left(\delta_{i j}-\frac{r_{i} r_{j}}{r^{2}}\right)\left(C_{i j}(0)-C_{i j}(\boldsymbol{r})\right) \\
D_{\theta \|}^{(3)}(r)=-6 \frac{r_{i} r_{j} r_{m}}{r^{3}} C_{i j m}(\boldsymbol{r}) .
\end{array}
$$

The unknown quantities $C_{i j}(\boldsymbol{r})$ and $C_{i j m}(\boldsymbol{r})$ determine thus the second- and third-order structure functions. These quantities will be determined in section 4 and the appendices, using techniques of the Direct Interaction Approximation type.

\subsection{Wavenumber spectra}

DIA is most conveniently defined in Fourier-space so that we introduce the Fourier-transforms of the above correlation-functions,

$$
\begin{aligned}
\Psi_{i j}(\boldsymbol{k}) & =\mathcal{F}\left[C_{i j}(\boldsymbol{r})\right]=\overline{\chi_{i}(\boldsymbol{k}) \chi_{j}(-\boldsymbol{k})} \\
\Xi_{i j m}(\boldsymbol{k}) & =\mathcal{F}\left[C_{i j m}(\boldsymbol{r})\right]=\overline{\chi_{i}(-\boldsymbol{k})\left[\chi_{j} \star \chi_{m}\right](\boldsymbol{k})},
\end{aligned}
$$

where $\mathcal{F}$ indicates a Fourier transform, $\chi_{i}(\boldsymbol{k})=\mathcal{F}\left[\chi_{i}(\boldsymbol{x})\right]$, and $\star$ denotes a convolution. Expressions (26) become then

$$
\begin{aligned}
D_{\theta \|}^{(2)}(r) & =2 \frac{r_{i} r_{j}}{r^{2}} \int \Psi_{i j}(\boldsymbol{k})\left[1-e^{i \boldsymbol{k} \cdot \boldsymbol{r}}\right] d \boldsymbol{k} \\
D_{\theta \perp}^{(2)}(r) & =\left(\delta_{i j}-\frac{r_{i} r_{j}}{r^{2}}\right) \int \Psi_{i j}(\boldsymbol{k})\left[1-e^{i \boldsymbol{k} \cdot \boldsymbol{r}}\right] d \boldsymbol{k} \\
D_{\theta \|}^{(3)}(r) & =-6 \frac{r_{i} r_{j} r_{m}}{r^{3}} \int \Xi_{i j m}(\boldsymbol{k}) e^{i \boldsymbol{k} \cdot \boldsymbol{r}} d \boldsymbol{k} .
\end{aligned}
$$

In order to simplify these expressions, we will express them using symmetry arguments (see for example the discussion on tensorial representation in [23]. The general form of the mirror-symmetric isotropic tensor $\Psi_{i j}(\boldsymbol{k})$ only depends on $k_{i}$ and $\delta_{i j}$ and we can therefore write it as

$$
\Psi_{i j}(\boldsymbol{k})=P_{i j}(\boldsymbol{k}) \frac{E^{s}(k)}{4 \pi k^{2}}+\Pi_{i j}(\boldsymbol{k}) \frac{E^{c}(k)}{4 \pi k^{2}} .
$$


with

$$
\begin{array}{r}
P_{i j}(\boldsymbol{k})=\delta_{i j}-\frac{k_{i} k_{j}}{k^{2}} \\
\Pi_{i j}(\boldsymbol{k})=\frac{k_{i} k_{j}}{k^{2}} .
\end{array}
$$

In this description, $E^{s}(k)$ is associated to the variance distribution of the solenoidal part of the vector field and $E^{c}(k)$ to the compressible (or potential) part. Similarly, $\Xi_{i j m}(\boldsymbol{k})$ can be written as

$$
\Xi_{i j m}(\boldsymbol{k})=\Xi^{I}(k) P_{m i j}(\boldsymbol{k})+\Xi^{I I}(k) k_{m} P_{i j}(\boldsymbol{k})+\Xi^{I I}(k) k_{m} \Pi_{i j}(\boldsymbol{k})
$$

where $P_{m i j}(\boldsymbol{k})=k_{i} P_{m j}+k_{j} P_{m i}$ and where we used that $\Xi_{i j m}(\boldsymbol{k})$ should be symmetric in the indices $i, j$. This implies for the spectra $\Xi^{I}(k), \Xi^{I I}(k)$ and $\Xi^{I I I}(k)$,

$$
\begin{aligned}
\Xi^{I}(k) & =\frac{1}{4 k^{2}} P_{m i j}(\boldsymbol{k}) \Xi_{i j m}(\boldsymbol{k}) \\
\Xi^{I I}(k) & =\frac{1}{2 k^{2}} k_{m} P_{i j}(\boldsymbol{k}) \Xi_{i j m}(\boldsymbol{k}) \\
\Xi^{I I I}(k) & =\frac{1}{k^{2}} k_{m} \Pi_{i j}(\boldsymbol{k}) \Xi_{i j m}(\boldsymbol{k}) .
\end{aligned}
$$

We further introduce the velocity-displacement correlation

$$
F_{i j}(\boldsymbol{k}) \delta\left(\boldsymbol{k}+\boldsymbol{k}^{\prime}\right)=\overline{u_{i}(\boldsymbol{k}) \chi_{j}\left(\boldsymbol{k}^{\prime}\right)},
$$

which can be expressed in an isotropic flow as

$$
F_{i j}(\boldsymbol{k})=P_{i j}(\boldsymbol{k}) \frac{F(k)}{4 \pi k^{2}} .
$$

Since the scalar corresponds to one component of the displacement vector-field, the scalar spectrum and scalar flux spectrum are related to $\Psi_{i j}$ and $F_{i j}$ by the following relations:

$$
\begin{array}{r}
F_{u \theta}(k)=\frac{1}{3} F(k) \\
E_{\theta}(k)=\frac{2}{3} E^{s}(k)+\frac{1}{3} E^{c}(k) .
\end{array}
$$

Substituting (30) and (32) into expressions (29), one obtains the following relations for the second- and third-order structure functions,

$$
\begin{aligned}
D_{\theta \|}^{(2)}(r) & =\frac{2}{3} \overline{\chi_{i} \chi_{i}}-\int E^{s}(k) f(k r) d k-\int E^{c}(k) g(k r) d k \\
D_{\theta \perp}^{(2)}(r) & =\frac{2}{3} \overline{\chi_{i} \chi_{i}}-\frac{1}{2} \int E^{s}(k) h(k r) d k-\frac{1}{2} \int E^{c}(k) f(k r) d k \\
D_{\theta \|}^{(3)}(r) & =-12 \pi \int k^{3}\left[j(k r)\left(2 \Xi^{I}(k)+\Xi^{I I}(k)\right)+l(k r) \Xi^{I I I}(k)\right] d k
\end{aligned}
$$


with

$$
\begin{array}{r}
f(x)=-4 \frac{x \cos (x)-\sin (x)}{x^{3}} \\
g(x)=2 \frac{2 x \cos (x)+x^{2} \sin (x)-2 \sin (x)}{x^{3}} \\
h(x)=4 \frac{x^{2} \sin (x)+x \cos (x)-\sin (x)}{x^{3}} \\
j(x)=-4 i \frac{-3 \sin (x)+x^{2} \sin (x)+3 x \cos (x)}{x^{5}} \\
l(x)=-2 i \frac{x^{3} \cos (x)+6 \sin (x)-6 x \cos (x)-3 x^{2} \sin (x)}{x^{5}} .
\end{array}
$$

The derivation of these relations roughly follows the lines in [24]. Up to here, the derivation was purely kinematic and the expressions in this section are exact. The second- and third-order structure functions are expressed as functions of the scalar wave-number spectra $E^{s}(k), E^{c}(k), \Xi^{I}(k), \Xi^{I I}(k)$ and $\Xi^{I I I}(k)$. These will be determined in section 4 .

The anisotropic character of the scalar correlations can be made more explicit by the following relation:

$$
E_{\theta}(k)=\int_{0}^{2 \pi} \int_{0}^{\pi} \Psi_{33}(\boldsymbol{k}) k^{2} \sin \theta d \theta d \phi .
$$

For convenience the angle $\theta$ is defined with respect to the direction of the mean scalar gradient and we introduce $\mu=\cos \theta$. The anisotropic spectrum characterizing the scalar variance is thus $\Psi_{33}(\boldsymbol{k})$, which can be written, using (30) as

$$
\Psi_{33}(\boldsymbol{k})(k, \mu)=\frac{1}{4 \pi k^{2}}\left(\left(1-\mu^{2}\right) E^{s}(k)+\mu^{2} E^{c}(k)\right)
$$

so that the isotropic part of the scalar spectrum is given by $E^{s}(k)$ and the anisotropic part is fully described by the quantity $E^{c}(k)-E^{s}(k)$. Similarly the velocity-scalar correlation is determined by $F_{33}(k)$, that can be written with (35) as

$$
F_{33}(\boldsymbol{k})(k, \mu)=\frac{1}{4 \pi k^{2}}\left(1-\mu^{2}\right) F(k) .
$$

For the two-point triple correlation, expression (25), we see that it is given by two scalar functions of the wavenumber: $\Xi^{I I I}(k)$ and $\left(2 \Xi^{I}(k)+\Xi^{I I}(k)\right)$,

$$
\Xi_{333}(\boldsymbol{k})=\left(2 \Xi^{I}(k)+\Xi^{I I}(k)\right) \mu\left(1-\mu^{2}\right)+\Xi^{I I I}(k) \mu^{3} .
$$

The description of the passive scalar fluctuations as a component of the isotropic displacement vector is hereby related to other approaches. For instance, expressions (44) and (45) can be obtained if the scalar spectrum is expanded as a sum of Legendre polynomials [25], as was done for the scalar spectrum and cospectrum by [26] and [27]. Also, a link can be made with the $\mathrm{SO}(3)$ decomposition $[28,27]$. We think that the use of the scalar displacement field helps to obtain a better intuition of the character of the anisotropy in the present case, since the field is isotropic and has a physical interpretation. 


\subsection{Scalar gradients}

The scalar gradient statistics can be obtained by taking the limit,

$$
\begin{gathered}
\overline{\left(\frac{\partial \theta}{\partial x_{\|}}\right)^{2}}=\lim _{r \rightarrow 0} S_{\|}^{(2)}(r) / r^{2} \\
\overline{\left(\frac{\partial \theta}{\partial x_{\perp}}\right)^{2}}=\lim _{r \rightarrow 0} S_{\perp}^{(2)}(r) / r^{2}
\end{gathered}
$$

These limits can be determined analytically by developing $f(x)$ and $g(x)$ and $h(x)$ as a Taylor series for small $x$, leading to

$$
\begin{aligned}
& \overline{\left(\frac{\partial \theta}{\partial x_{\|}}\right)^{2}}=\frac{1}{15} \int 2 k^{2} E^{s}(k)+3 k^{2} E^{c}(k) d k \\
& \overline{\left(\frac{\partial \theta}{\partial x_{\perp}}\right)^{2}}=\frac{1}{15} \int 4 k^{2} E^{s}(k)+k^{2} E^{c}(k) d k
\end{aligned}
$$

The ratio of the variance of the gradients in the two directions is then given by

$$
\frac{\overline{\left(\frac{\partial \theta}{\partial x_{\|}}\right)^{2}}}{\overline{\left(\frac{\partial \theta}{\partial x_{\perp}}\right)^{2}}}=1+\frac{2 \int k^{2}\left(E^{c}(k)-E^{s}(k)\right) d k}{\int k^{2}\left(4 E^{s}(k)+E^{c}(k)\right) d k} .
$$

This ratio, which measures the small-scale isotropy, tends thus to unity (indicating isotropy) if the spectra $E^{c}(k)$ and $E^{s}(k)$ are close enough.

\section{Closed expressions for the second-order and third-order correlations}

The starting point to obtain closed expressions is the equation for $\chi_{i}$, expression (23), which we write in Fourier space.

$$
\left[\partial_{t}+D k^{2}\right] \chi_{i}(\boldsymbol{k})=-i k_{m}\left[u_{m} \star \chi_{i}\right](\boldsymbol{k})+u_{i}(\boldsymbol{k}) .
$$

The equation for $\Psi_{i j}(\boldsymbol{k})$ can be derived from this equation by multiplying (23) by $\chi_{j}\left(\boldsymbol{k}^{\prime}\right)$, then multiplying the equation for $\chi_{j}\left(\boldsymbol{k}^{\prime}\right)$ by $\chi_{i}(\boldsymbol{k})$, and averaging the sum of the two equations and invoking homogeneity. The resulting expression is

$$
\left[\partial_{t}+2 D k^{2}\right] \Psi_{i j}(\boldsymbol{k})=T_{i j}(\boldsymbol{k})+2 F_{i j}(\boldsymbol{k})
$$

with

$$
T_{i j}(\boldsymbol{k})=-2 i k_{m} \iint \delta(\boldsymbol{k}-\boldsymbol{p}-\boldsymbol{q}) \overline{u_{m}(\boldsymbol{p}) \chi_{i}(\boldsymbol{q}) \chi_{j}(-\boldsymbol{k})} d \boldsymbol{p} d \boldsymbol{q}
$$


It is now possible to derive the evolution equation for $E^{s}(k)$ and $E^{c}(k)$ by multiplying (53) by either the operators $P_{i j}$ or $\Pi_{i j}$. This yields

$$
\begin{aligned}
& {\left[\partial_{t}+2 D k^{2}\right] E^{s}(k)=2 \pi k^{2} P_{i j}(\boldsymbol{k}) T_{i j}(\boldsymbol{k})+2 F(k)} \\
& {\left[\partial_{t}+2 D k^{2}\right] E^{c}(k)=4 \pi k^{2} \Pi_{i j}(\boldsymbol{k}) T_{i j}(\boldsymbol{k}) .}
\end{aligned}
$$

We see that the production term, proportional to $F(k)$ only appears in the solenoidal part. The unclosed term $T_{i j}(\boldsymbol{k})$ is evaluated using the Eulerian DIA technique which is subsequently simplified by assuming exponential timedependence of two-time correlations, with a carefully determined Lagrangian timescale, rendering it in agreement with Lagrangian theories such as the Lagrangian History DIA [29] or the Lagrangian Renormalized Approximation [30]. The procedure is outlined in detail in section 3 of [22], of which a summary is presented in appendix A. The resulting expression is given in appendix B. The closed expressions for $\Xi^{I}(k), \Xi^{I I}(k)$ and $\Xi^{I I I}(k)$ are given in appendix $\mathrm{C}$

The closed expression for $T_{i j}(\boldsymbol{k})$ allows to solve numerically the evolution equations for $E^{s}(k)$ and $E^{c}(k)$, expressions (55), and these are integrated together with the equation for $E(k)$ and $F(k)$

$$
\begin{aligned}
{\left[\partial_{t}+2 \nu k^{2}\right] E(k) } & =T^{E}(k)+\mathcal{I}_{E}(k) \\
{\left[\partial_{t}+(D+\nu) k^{2}\right] F(k) } & =T^{F}(k)+\mathcal{I}_{F}(k) .
\end{aligned}
$$

The exact expressions for $T^{E}(k)$ and $T^{F}(k)$ are derived from the Navier-Stokes equations,

$$
\left[\partial_{t}+\nu k^{2}\right] u_{i}(\boldsymbol{k})=-\frac{i}{2} P_{i j m}(\boldsymbol{k})\left[u_{j} \star u_{m}\right](\boldsymbol{k})+f_{i}(\boldsymbol{k}) .
$$

where

$$
f_{i}(\boldsymbol{k})=\frac{u_{i}(\boldsymbol{k})}{2 E(k)} H\left(k_{f}-k\right)
$$

with $H(x)$ the Heaviside function and $k_{f}$ the wavenumber below which the forcing is active. This forcing yields, in the equations (57) and (58) the following forcing terms:

$$
\begin{array}{r}
\mathcal{I}_{E}(k)=H\left(k_{f}-k\right) \\
\mathcal{I}_{F}(k)=H\left(k_{f}-k\right) \frac{F(k)}{2 E(k)} .
\end{array}
$$

These equations are closed using the Lagrangian Markovianized Field Approximation (LMFA, [31], 2013), having similarities with the Test-Field Model [32], and expressions are given in appendix D.

\section{Choice of the timescales}

The choice of the timescales is, perhaps, the most delicate issue in the use of triadic closures, in particular when the time-history is involved in the closure, as is 
the case in the DIA. In order to obtain statistics in agreement with KolmogorovCorrsin-Obukhov phenomenology, one needs to use timescales which are compatible with the Lagrangian character of the time-correlations. An incorrect choice of the reference frame can completely change the resulting statistics [33]. In Markovian closures, such as the Eddy-Damped Quasi-Normal Markovian (EDQNM) closure or the LMFA closure used here, the Lagrangian character must be taken into account in the timescale governing the triad-interactions. This triad-time is intimately related to the time a fluid or scalar structure of a certain size remains correlated to a fluid trajectory. We will first show how these timescales appear in the general structure of the closed equations. Then, by dimensional and physical arguments, we will illustrate how the choice of these timescales affects the scaling behavior of the different wavenumber spectra in a long inertial range.

The evolution of $E(k)$ is given by the Lin-equation (57), in which the nonlinear transfer is closed by a closure theory. The resulting expression is of the form

$$
T^{E}(k)=\iint \Theta^{E}(k, p, q) f[E(k), E(p), E(q), \boldsymbol{k}, \boldsymbol{p}, \boldsymbol{q}] d \boldsymbol{p} d \boldsymbol{q} .
$$

We see that the integrand of this equation consists of the product of the triadtimescale $\Theta^{E}(k, p, q)$ with a function of wavevectors and energy spectra. Clearly, a large value of $\Theta^{E}(k, p, q)$ will increase the transfer $T^{E}(k)$ and a small value will decrease it.

Similarly, the nonlinear term of the equation for $F(k)$ is given by

$$
T^{F}(k)=\iint \Theta^{F}(k, p, q) f[E(k), E(p), E(q), F(k), F(p), F(q), \boldsymbol{k}, \boldsymbol{p}, \boldsymbol{q}] d \boldsymbol{p} d \boldsymbol{q} \text {. }
$$

The dynamics of $E^{s}(k)$ and $E^{c}(k)$ are given by the transfer $T^{s}(k)$ and $T^{c}(k)$, which are both of the form

$$
\begin{aligned}
T^{s, c}(k)= & \iint \Theta^{s, c}(k, p, q) f[E(k), E(p), E(q), \\
& \left.E^{s}(k), E^{c}(k), E^{s}(p), E^{c}(p), E^{s}(q), E^{c}(q), F(k), F(p), F(q), \boldsymbol{k}, \boldsymbol{p}, \boldsymbol{q}\right] d \boldsymbol{p} d \boldsymbol{q} .
\end{aligned}
$$

Finally, $\Xi^{I}(k), \Xi^{I I}(k)$ and $\Xi^{I I I}(k)$ are of the form

$$
\begin{gathered}
\Xi^{I}(k)=\iint \Theta^{\Xi}(k, p, q) f\left[E^{s}(k), E^{c}(k), E^{s}(p),\right. \\
\left.E^{c}(p), E^{s}(q), E^{c}(q), F(k), F(p), F(q), \boldsymbol{k}, \boldsymbol{p}, \boldsymbol{q}\right] d \boldsymbol{p} d \boldsymbol{q},
\end{gathered}
$$

in which the timescales $\Theta^{E}(k), \Theta^{F}(k), \Theta^{s, c}(k, p, q)$ and $\Theta^{\Xi}(k, p, q)$, in the long- 
time limit, are given by

$$
\begin{array}{r}
\Theta^{E}(k, p, q)=\frac{1}{\eta(k)+\eta(p)+\eta(q)} \\
\Theta^{F}(k, p, q)=\frac{1}{\eta_{\theta}(k)+\eta(p)+\eta(q)} \\
\Theta^{s, c}(k, p, q)=\frac{1}{\eta_{\theta}(k)+\eta(p)+\eta_{\theta}(q)} \\
\Theta^{\Xi}(k, p, q)=\frac{1}{\eta_{\theta}(k)+\eta_{\theta}(p)+\eta_{\theta}(q)} .
\end{array}
$$

This precise form appears though the assumption of an exponential time-dependence of the two-time correlations plus a fluctuation-dissipation theorem for the Lagrangian velocity and scalar correlations.

The timescale $\eta(k)^{-1}$ is determined through a procedure outlined in [31] and behaves in the inertial range proportional to $\epsilon^{-1 / 3} k^{-2 / 3}$. This scaling is rather well-accepted in the field of turbulence research. The choice of $\eta_{\theta}(k)$ is less well documented. We will focus on two possibilities: first $\eta_{\theta}(k) \sim \mathcal{T}^{-1} \sim \epsilon^{1 / 3} k_{0}^{2 / 3}$, independent of the wavenumber and with $k_{0}$ corresponding to the energy containing range of the spectrum second, $\eta_{\theta}(k) \sim \eta(k)$ in the inertial range. If we consider $k, p, q$ all in the inertial range and comparable in magnitude: $p=\alpha k$, $q=\beta k$, with $\alpha, \beta=\mathcal{O}(1)$ we have

$$
\begin{array}{r}
\Theta^{E}(k, p, q)=\frac{k^{-2 / 3}}{\epsilon^{1 / 3}\left(1+\alpha^{2 / 3}+\beta^{2 / 3}\right)} \\
\Theta^{F}(k, p, q) \approx \frac{k^{-2 / 3}}{\epsilon^{1 / 3}\left(\alpha^{2 / 3}+\beta^{2 / 3}\right)} \\
\Theta^{s}(k, p, q) \approx \frac{k^{-2 / 3}}{\epsilon^{1 / 3} \beta^{2 / 3}} \\
\Theta^{\Xi}(k, p, q)=\frac{1}{3 \epsilon^{1 / 3} k_{0}^{2 / 3}},
\end{array}
$$

which shows that,

$$
\begin{array}{r}
\Theta^{E}(k, p, q) \sim \Theta^{F}(k, p, q) \sim \Theta^{s}(k, p, q) \sim \epsilon^{-1 / 3} k^{-2 / 3} \\
\Theta^{\Xi}(k, p, q) \sim k^{0} .
\end{array}
$$

We see that, apart from $\Theta^{\Xi}(k, p, q)$, all triad timescales are proportional to $k^{-2 / 3}$. In the case in which $\eta_{\theta}(k) \sim \eta(k)$, we have,

$$
\Theta^{E}(k, p, q) \sim \Theta^{F}(k, p, q) \sim \Theta^{s}(k, p, q) \sim \Theta^{\Xi}(k, p, q) \sim k^{-2 / 3},
$$

and for this choice $\Theta^{\Xi}(k, p, q)$ behaves similar to the other three triad times. This shows that the choice of the $\eta_{\theta}(k)$ in the inertial range changes the scaling of $\Theta^{\Xi}(k, p, q)$, but does not influence the scaling of the other triad times (but 
changes their magnitude). This is an important observation, because it shows how the scaling of the third-order scalar structure function is independent of the scaling of the second-order structure function. We will show in the following that this can lead to an anomalous value of the scalar gradient skewness, while the second-order structure functions scale in agreement with Corrsin-Obukhov phenomenology.

In the following sections we will use

$$
\eta(k)=\frac{E(k)}{F^{0}(k)}+\nu k^{2},
$$

where $F^{0}(k)$ is the velocity-displacement correlation spectrum for a value of zero diffusivity. This frequency is proportional to $\epsilon^{1 / 3} k^{2 / 3}$ in the inertial range and to $\nu k^{2}$ in the dissipation range. For the triad-frequency corresponding to the scalar field we choose

$$
\eta_{\theta}(k)=\mathcal{T}^{-1},
$$

where the integral timescale is defined as

$$
\mathcal{T}=\frac{9 \pi}{16} \frac{\int k^{-1} E(k) d k}{\left(\int E(k) d k\right)^{3 / 2}} .
$$

This choice, $\eta_{\theta}(k)=\mathcal{T}^{-1}$, can be justified phenomenologically by considering the Lagrangian DIA closure for linearly forced Burgers' turbulence [34],

$$
\partial_{t} u+u \partial_{x} u=\alpha u
$$

According to Lagrangian DIA, in the case of $\alpha=0$ the timescale governing the dynamics is proportional to the entire time-history of the flow. In other words, following a Lagrangian trajectory, the velocity does not decorrelate. However, if $\alpha>0$, the asymptotic limit of the timescale is $\alpha^{-1}$, corresponding to the time it takes for a fluid particle to travel between two shocks. In the case of the passive scalar, the production term on the righthandside of equation (3) can be argued to play the same role as the forcing term on the righthandside of (73), and it is perhaps not fortuitous that both Burgers' equation and the scalar fluctuations in the presence of a mean gradient give birth to ramp-cliff structures (generally called shocks in the case of Burgers' turbulence). Indeed, these ramp-cliff structures play the same role for the scalar as the shocks play for the Burgers velocity field. Once a fluid particle passes through a ramp-cliff structure, the scalar will decorrelate from the trajectory. Therefore, whereas in isotropic scalar turbulence the scalar fluctuations will remain correlated to a trajectory over a timescale of the order of the diffusive time [29], in the presence of a mean scalar gradient the correlation will be limited by the time it takes the flow to build up a ramp-cliff structure.

A further study on the similarity between the dynamics of Burgers' turbulence and the scalar in the presence of a mean gradient is currently in progress. 

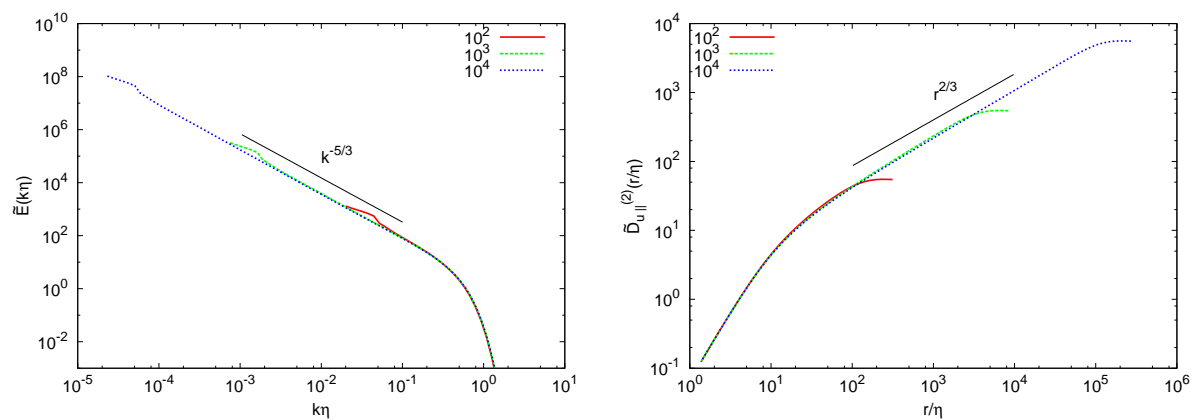

Figure 1: Left: results for the normalized energy spectrum $\tilde{E}(k \eta)=$ $E(k \eta) \nu^{-5 / 4} \epsilon^{-1 / 4}$. Right: results for the normalized second-order longitudinal structure function $\tilde{D}_{u}^{(2)}(r / \eta)$. All results are shown for the Reynolds numbers $R_{\lambda}=10^{2}, 10^{3}, 10^{4}$.

The choice (71) is essential to obtain agreement with experimental and numerical observations of the scalar gradient skewness. To illustrate this we will also, in section 6.4 present results for the choice $\eta_{\theta}(k)=\eta(k)$, to determine the sensitivity of the results on the choice of the timescale.

\section{$6 \quad$ Numerical Results}

Equations (55), (56), (57) and (58) are integrated numerically on a logarithmically spaced grid with 15 wavenumbers per decade for Taylor-scale Reynolds numbers $R_{\lambda}=10^{2}, 10^{3}, 10^{4}$. Due to the presence of the large scale forcing (61), with $k_{f}=2.5$, the system tends to a steady state in which the energy input is balanced by the viscous dissipation. It is during this steady state that all the statistics are evaluated. The wavenumber spectra and structure functions are all plotted in normalized units as discussed in section 2.2.

\subsection{Results for the velocity field}

In Figure 1, left, we show the energy spectra $\tilde{E}(k \eta)$, normalized by Kolmogorov variables, for three different Reynolds numbers, $R_{\lambda}=10^{2}, 10^{3}$ and $10^{4}$. In the following all results will be presented for these three cases. The spectra perfectly collapse, as expected. In Figure 1, right, we show the second-order structure functions for longitudinal velocity fluctuations, again normalized by Kolmogorov variables, for three different Reynolds numbers, $R_{\lambda}=10^{2}, 10^{3}$ and $10^{4}$. A clear scaling proportional to $r^{2 / 3}$ is observed and the small scales collapse in Kolmogorov variables.

In Figure 2, left, we show the third-order structure functions and in Figure 2, right, the velocity increment skewness. These quantities have been discussed in detail in [35] as obtained from EDQNM computations, and we will not discuss 

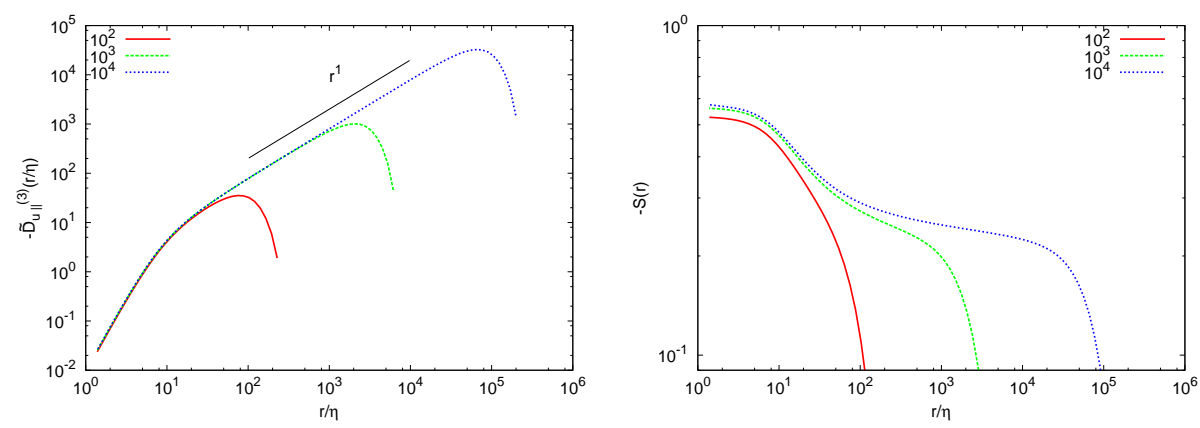

Figure 2: Left: normalized third-order longitudinal structure function, $\tilde{D}_{u}^{(3)}(r / \eta)$. Right: longitudinal increment skewness $-S(r / \eta)$.

them here further. All we say here is that the expected scaling is observed, but that for the velocity increment skewness a very large Reynolds number is needed to observe asymptotic scaling.

\subsection{Scalar Wavenumber spectra}

In Figure 3 we show the results for the spectra $E^{s}(k)$ and $E^{c}(k)$. Both spectra scale proportional to $k^{-5 / 3}$ and the difference between the two spectra is small. Only in the smallest wavenumbers a difference between the two spectra is observed. The collapse in the dissipation range is almost perfect. As we will see in section 6.3 , this shows that the small scale anisotropy, as measured by second-order quantities, is small.

In Figure 4, We show the scalar spectrum $E_{\theta}(k) \equiv 2 E^{s}(k)+E^{c}(k)$. Since $E^{s}(k)$ and $E^{c}(k)$ showed very similar behavior, it is not surprising that $E_{\theta}(k)$ is qualitatively identical to them. Again a clear $k^{-5 / 3}$ scaling shows the agreement with Corrsin-Obukhov phenomenology. In Figure 4, right, we show the behaviour of the scalar flux spectrum. As in previous studies [36, 37, 38], a clear $k^{-7 / 3}$ scaling is observed, confirming the dimensional arguments by Lumley, discussed in section 2.2 .

\subsection{Scalar increment statistics and anisotropy}

In Figure 5 we show the second-order scalar structure functions for separation vectors parallel with and perpendicular to the direction of the mean scalar gradient. The behaviour of both is very similar. A clear $r^{2 / 3}$ scaling range is observed. The normalization allows to collapse the graphs almost perfectly for the smallest separation distances. At these values of $r / \eta$, the structure functions are proportional to $r^{2}$, as is expected since at these scales the scalar field becomes smooth, so that the increments scale proportional to $r$.

In Figure 6 we show the third-order scalar structure function. Two different normalizations are used. In Figure 6, left, the normalization proposed in expres- 


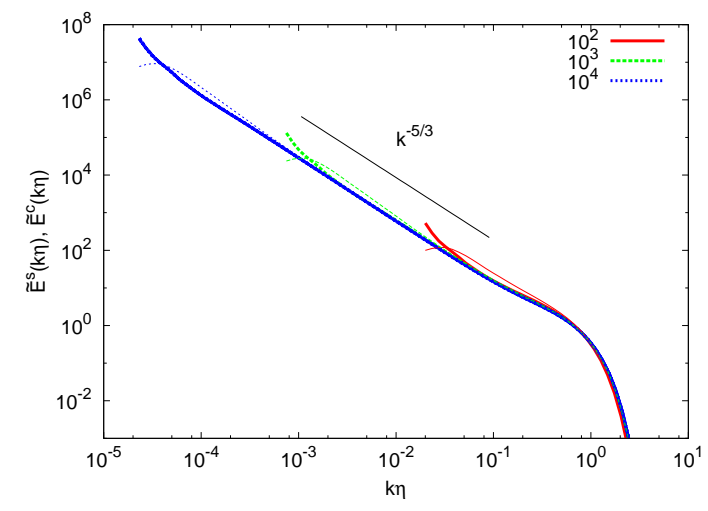

Figure 3: Wavenumber spectra of the solenoidal and compressible parts of the displacement vector $\tilde{E}^{s}(k \eta)$ (thick lines), $\tilde{E}^{c}(k \eta)$ (thin lines).
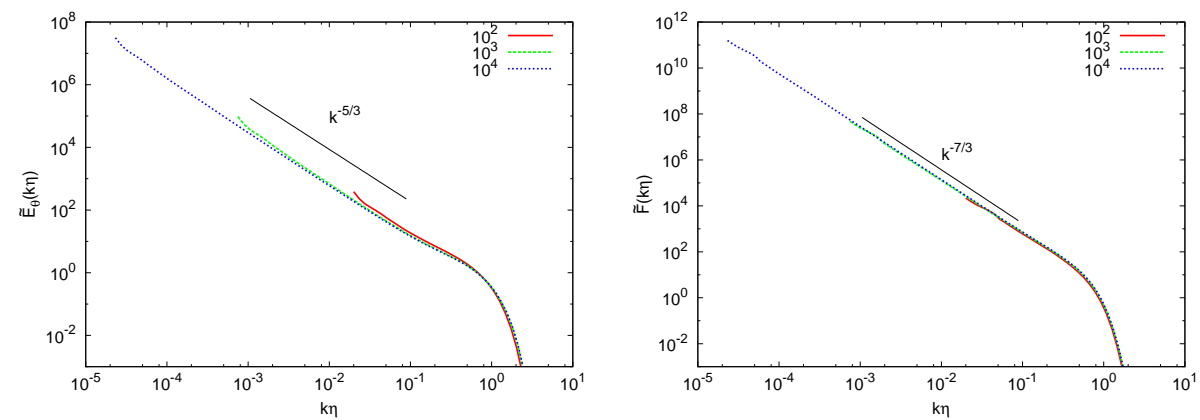

Figure 4: Left: scalar spectrum $\tilde{E}_{\theta}(k \eta)$. Right: scalar flux spectrum $\tilde{F}(k \eta)$.
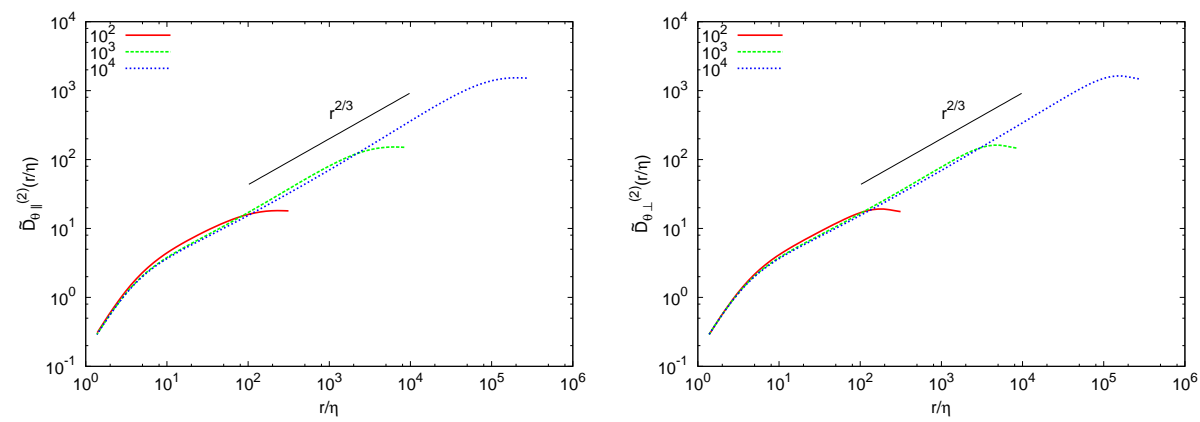

Figure 5: Left: parallel scalar velocity increments $D_{\theta \|}^{(2)}(r / \eta)$. Right: perpendicular scalar structure function $D_{\theta \perp}^{(2)}(r / \eta)$. 

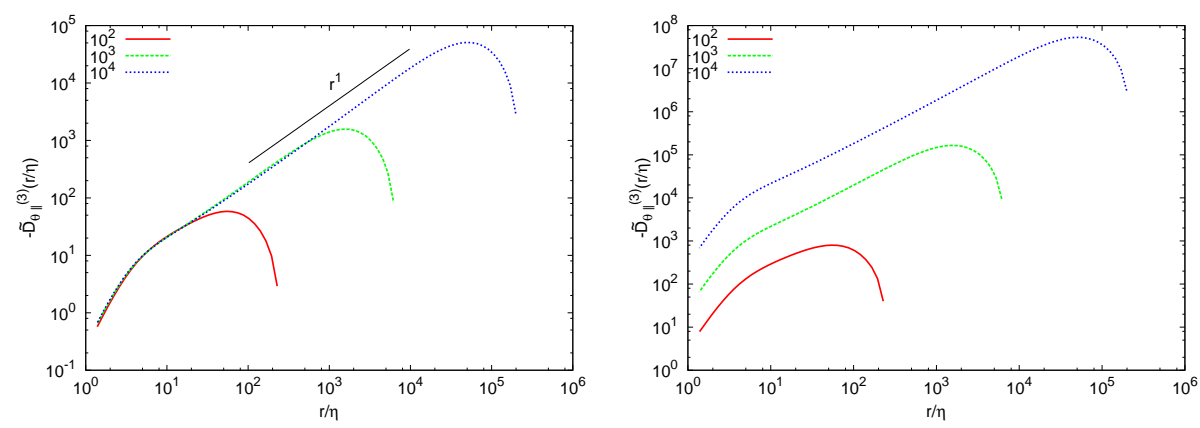

Figure 6: Left: third-order scalar structure function normalized as $\tilde{D}_{\theta \|}^{(3)}(r / \eta)=\left(\Gamma \epsilon_{\theta} \nu^{5 / 4} \epsilon^{-3 / 4}\right)^{-1} D_{\theta \|}^{(3)}(r / \eta)$. Right normalized as $\tilde{D}_{\theta \|}^{(3)}(r / \eta)=$ $\left(\Gamma \epsilon_{\theta} \mathcal{T} \nu^{3 / 4} \epsilon^{-1 / 4}\right)^{-1} D_{\theta \|}^{(3)}(r / \eta)$.

sion (20) is used. In Figure 6, right, we use the normalization (76) which we will suggest in section 7 . This latter normalization contains the integral timescale, which we used in the definition of the triad-correlation timescale in section 5 . Clearly, this latter normalization collapses the results much better. The scaling in the inertial range is proportional to $r$.

To quantify the anisotropy of the scalar field, we show in Figure 7, left, the ratio of the parallel and the perpendicular scalar structure functions. The value is not far from unity. Even in the largest scales, the anisotropy is under 10\% as measured by this ratio. In the smallest scales the ratio tends to unity, except for the lowest Reynolds number, where a small anisotropy of a few percent is measured. According to these results, Corrsin-Obukhov phenomenology seems to hold approximately.

The scalar gradient skewness is shown in Figure 7, right. An approximate plateau is observed in the inertial range, also observed in experiments, and the small scale limit tends to a value of approximately $-S_{\theta}(r) \approx 2$, this limit gives the value of the scalar-gradient skewness, which does saturate at a non-zero value.

The Reynolds number dependence of the ratio of the mean-square parallel scalar gradients to the mean-square perpendicular gradients is displayed in Figure 8, left. This quantity corresponds to the small $r$ limit of Figure 7 . The ratio tends rapidly to unity. This tendency can be explained from the results for the spectra $E^{s}(k)$ and $E^{c}(k)$, in Figure 3 and relation (51).

In Figure 8, right we show the parallel gradient skewness. This quantity roughly remains constant as a function of the Reynolds number, indicating a small scale anisotropy for the third-order correlations. It seems that small scale isotropy of second-order scalar increments is independent of the persistence of the scalar gradient skewness. 

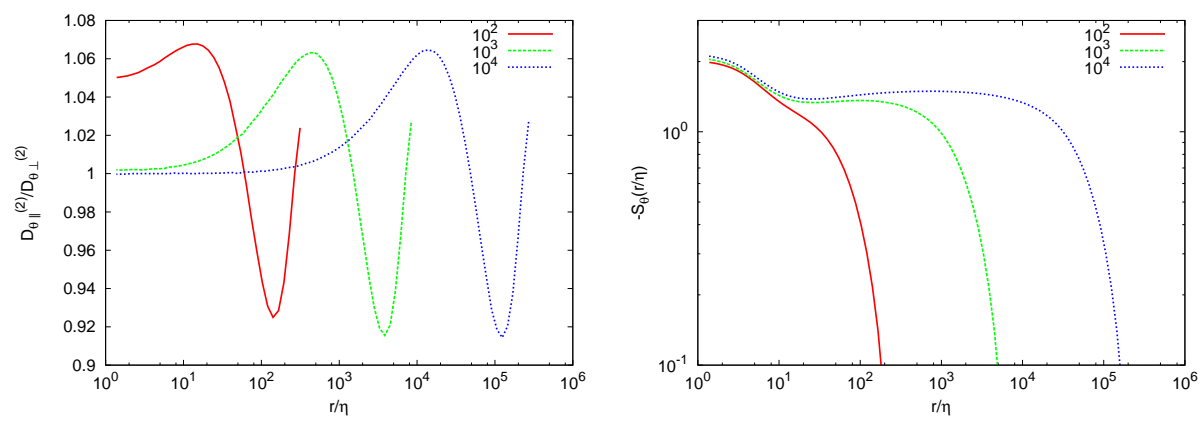

Figure 7: Left: ratio of the parallel and perpendicular secon-order scalar structure functions. Right: scalar increment skewness $-S_{\theta}(r / \eta)$.
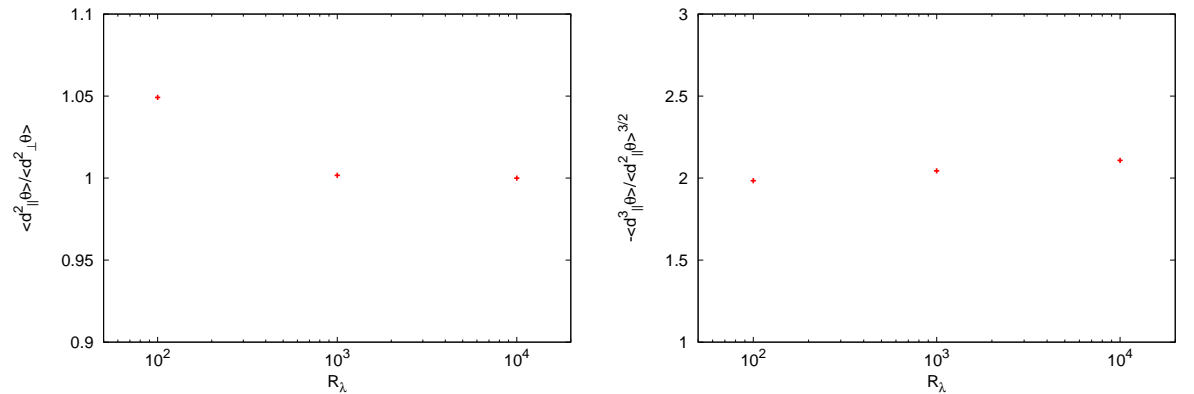

Figure 8: Left: Reynolds number dependence of the ratio of the mean-square parallel scalar gradients to the mean-square perpendicular gradients. Right: Reynolds number dependence of the parallel scalar gradient skewness. 

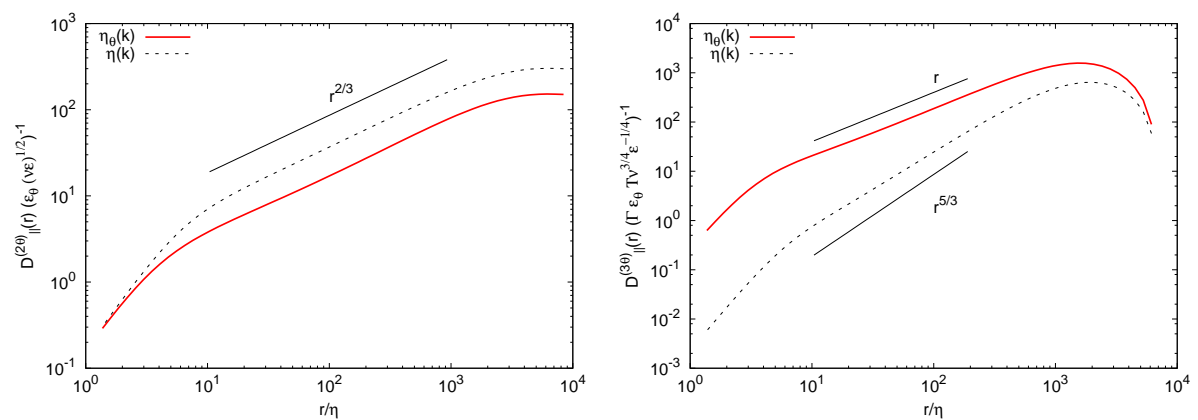

Figure 9: Influence of the timescale on the scaling of the second- (left) and third-order (right) scalar structure functions.

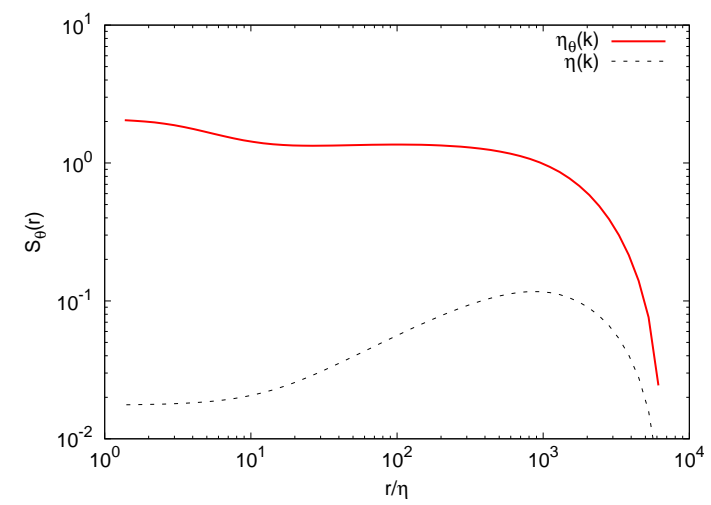

Figure 10: Influence of the timescale on the scaling of scalar increment skewness.

\subsection{Influence of the timescale}

We now show the influence of the scalar timescale on the results. In the previous section we used for the scalar timescale, $\eta_{\theta}(k)^{-1}=\mathcal{T}$. The difference with the results when $\eta_{\theta}(k)=\eta(k)$ used are shown in Figure 9 and 10. In Figure 9 we observe that when the timescale is changed the scaling of the second-order structure function does not change, as was anticipated in section 5. However, its magnitude does. The third-order structure function changes its scaling drastically. If the velocity timescale is used for the scalar, the scaling changes from $r^{1}$ to $r^{5 / 3}$. This implies also that the scalar increment skewness changes, as is illustrated in Figure 10. Clearly, to obtain agreement with scaling observed in experiments and simulations the scalar timescale should be proportional to the integral timescale or another timescale, independent of the wavenumber in the inertial range. 


\section{Discussion and conclusion}

The main result of the present investigation is the clear observation that secondand third-order scalar correlations are not governed by the same dynamics. Where the origin of this difference lies can be most clearly seen as follows. The convolution products in the Navier-Stokes and scalar equations most naturally introduce the interaction between three-wave modes, or triads. The triads which determine the dynamics of $E^{s}(k)$ and $E^{c}(k)$ (and thereby the scalar spectrum $E_{\theta}(k)$ and the second-order scalar structure functions) are however not the same as those which determine the functions $\Xi(k)$ (which determine the third-order scalar structure function). If we consider the dynamics of the scalar field, we see from equation (54) that the triads intervening in the dynamics of the secondorder quantities are of the form

$$
\tau_{i j m}(\boldsymbol{k}, \boldsymbol{p}, \boldsymbol{q})=\delta(\boldsymbol{k}-\boldsymbol{p}-\boldsymbol{q}) \overline{\chi_{i}(-\boldsymbol{k}) u_{j}(\boldsymbol{p}) \chi_{m}(\boldsymbol{q})} .
$$

For the dynamics of the third-order gradient correlations we see from (28) that the triads are

$$
\tau_{i j m}^{\prime}(\boldsymbol{k}, \boldsymbol{p}, \boldsymbol{q})=\delta(\boldsymbol{k}-\boldsymbol{p}-\boldsymbol{q}) \overline{\chi_{i}(-\boldsymbol{k}) \chi_{j}(\boldsymbol{p}) \chi_{m}(\boldsymbol{q})} .
$$

Since we consider the statistical average, these quantities are not simply triple products, but they are triple correlations. It is the correlation time of the triads which will determine the dynamics. It was shown by [33], that one should consider these time-correlations in a Lagrangian frame. Since the scalar, obviously, behaves differently from the velocity, the triad containing a velocity component does not decorrelate on the same timescale as the triad containing only scalar modes. As the scalar is passive, it will not decorrelate from a trajectory, except under the influence of diffusion, or by a forcing term in the equations, such as the $\boldsymbol{\Gamma} \cdot \boldsymbol{u}$ term in equation (3). In physical space one can understand the correlation-time of a scalar blob on a Lagrangian trajectory to be limited by the time it takes a fluid particle to encounter a ramp-cliff structure. This will obviously not change the correlation-time of the velocity, since the scalar is passive, but it will limit the correlation of the scalar in a similar way as shocks in Burgers' turbulence limit the Lagrangian correlation time of the velocity. In Navier-Stokes turbulence, the velocity will decorrelate not only through forcing terms or viscosity, but also through the influence of pressure [29]. These are the reasons that the Lagrangian correlation timescales associated with $\tau_{i j m}$ and $\tau_{i j m}^{\prime}$ are different and this is the reason for the persistent scalar gradient skewness.

The implication for the scaling of the third-order structure functions is that these are now proportional to the integral timescale. Dimensional analysis, imposing the third-order structure function to be proportional to the integral timescale $\mathcal{T}$ and the value of the mean scalar gradient $\Gamma$, leads then to the following scaling in the inertial range,

$$
D_{\theta \|}^{(3)}(r)=\Gamma \epsilon_{\theta} \mathcal{T} \nu^{3 / 4} \epsilon^{-1 / 4} \tilde{D}_{\theta \|}^{(3)}(r / \eta),
$$


and we have in the inertial range,

$$
D_{\theta \|}^{(3)}(r)=\Gamma \epsilon_{\theta} \mathcal{T} r .
$$

This scaling allowed to collapse the results in Figure 6. In experiments and DNS, this is also what is observed, which provides support for the results obtained, and assumptions made in the present investigation.

\section{A DIA procedure and single-time simplification}

The procedure we use to obtain closed expressions for the different triple correlations is related to the Eulerian DIA. A subsequent simplification allows to obtain single-time expressions which are compatible with K41 phenomenology if a Lagrangian correlation timescale is chosen. An outline of the procedure is here given. Both the velocity and the displacement variable are expanded around a state $u_{i}^{(0)}$ (and $\chi_{i}^{(0)}$ ) in which the mode-coupling of a particular triad $k, p, q$ is removed. The nonlinear coupling of this single mode, indicated by superscript (1) is then treated as a small perturbation on this state:

$$
\begin{gathered}
u_{i} \rightarrow u_{i}^{(0)}+u_{i}^{(1)} \\
\chi_{i} \rightarrow \chi_{i}^{(0)}+\chi_{i}^{(1)} .
\end{gathered}
$$

The influence of this perturbation through nonlinear coupling and viscosity and diffusion is then [39], [40]:

$$
\begin{aligned}
u_{j}^{(1)}(\boldsymbol{p}, t) & =-i P_{j a b}(\boldsymbol{p}) \int_{0}^{t} G(\boldsymbol{p}, t, s)\left[u_{a}^{(0)}(\boldsymbol{k}, s) u_{b}^{(0)}(-\boldsymbol{q}, s)\right] d s \\
\chi_{i}^{(1)}(-\boldsymbol{k}, t) & =i k_{a} \int_{0}^{t} G^{\chi}(\boldsymbol{k}, t, s)\left[u_{a}^{(0)}(-\boldsymbol{p}, s) \chi_{i}^{(0)}(-\boldsymbol{q}, s)+u_{a}^{(0)}(-\boldsymbol{q}, s) \chi_{i}^{(0)}(-\boldsymbol{p}, s)\right] d s,
\end{aligned}
$$

in which $G(\boldsymbol{k}, t, s)$ and $G^{\chi}(\boldsymbol{k}, t, s)$ are the response function of the velocity and displacement field, respectively. These response functions are here determined in a way different from Eulerian DIA. The expressions (79) are substituted in the triple correlations and the weak-dependence hypothesis allows to write the fourth order correlations as products of two-time second-order correlations such as $E(k, t, s) \delta\left(\boldsymbol{k}+\boldsymbol{k}^{\prime}\right)=2 \pi k^{2} \overline{u_{i}(\boldsymbol{k}, t) u_{i}\left(\boldsymbol{k}^{\prime}, s\right)}$

It is here, following [22], that we replace these quantities by their analogues 
defined on Lagrangian trajectories,

$$
\begin{aligned}
E(k, t, s) & \rightarrow E(k, t \mid s) \\
G(k, t, s) & \rightarrow G(k, t \mid s) \\
F(k, t, s) & \rightarrow F^{\prime}(k, t \mid s) \\
F(k, s, t) & \rightarrow F^{\prime \prime}(k, t \mid s) \\
E^{s}(k, t, s) & \rightarrow E^{s}(k, t \mid s) \\
E^{c}(k, t, s) & \rightarrow E^{c}(k, t \mid s),
\end{aligned}
$$

with

$$
\begin{gathered}
F^{\prime}(k, t \mid s) \delta\left(\boldsymbol{k}+\boldsymbol{k}^{\prime}\right)=2 \pi k^{2} \overline{u_{i}(\boldsymbol{k}, t) \chi_{i}\left(\boldsymbol{k}^{\prime}, t \mid s\right)} \\
F^{\prime \prime}(k, t \mid s) \delta\left(\boldsymbol{k}+\boldsymbol{k}^{\prime}\right)=2 \pi k^{2} \overline{u_{i}(\boldsymbol{k}, t \mid s) \chi_{i}\left(\boldsymbol{k}^{\prime}, t\right)}
\end{gathered}
$$

We use the further assumption that the two-time quantities decay exponentially in time by posing

$$
\begin{array}{r}
G(k, t \mid s)=H(t-s) \exp [-(t-s) \eta(k)] \\
G^{\chi}(k, t \mid s)=H(t-s) \exp \left[-(t-s) \eta^{\theta}(k)\right] \\
E(k, t \mid s)=E(k, t)[G(k, t \mid s)+G(k, s \mid t)] \\
F^{\prime}(k, t \mid s)=F(k, t)[G(k, s \mid t)+G(k, t \mid s)] \\
F^{\prime \prime}(k, t \mid s)=F(k, t)\left[G^{\chi}(k, s \mid t)+G^{\chi}(k, t \mid s)\right] \\
E^{s}(k, t \mid s)=E(k, t)\left[G^{\chi}(k, t \mid s)+G^{\chi}(k, s \mid t)\right] \\
E^{c}(k, t \mid s)=E(k, t)\left[G^{\chi}(k, t \mid s)+G^{\chi}(k, s \mid t)\right]
\end{array}
$$

so that

$$
\int_{-\infty}^{t} d s G(p, t \mid s) E(q, t \mid s) F^{\prime \prime}(k, t \mid s)=\Theta^{F}(k p q) E(q, t) F(k, t)
$$

with

$$
\Theta^{F}(k p q)=\frac{1}{\eta^{\theta}(k)+\eta(p)+\eta(q)},
$$

in which $\eta^{\theta}(k)$ and $\eta(k)$ need to be determined. This procedure allows to obtain closed expressions for all correlations containing the velocity and displacement vector. Further details can be found in [22].

\section{B A model for the transfer of the advected vec- tor field}

The DIA approximation of $T_{i j}(\boldsymbol{k})$ is given by

$$
\begin{aligned}
& T_{i j}(\boldsymbol{k})=-2 i k_{m} \iint \delta(\boldsymbol{k}-\boldsymbol{p}-\boldsymbol{q})[ \\
& \left.\overline{u_{m}(\boldsymbol{p})^{(1)} \chi_{i}(\boldsymbol{q}) \chi_{j}(-\boldsymbol{k})}+\overline{u_{m}(\boldsymbol{p}) \chi_{i}(\boldsymbol{q})^{(1)} \chi_{j}(-\boldsymbol{k})}+\overline{u_{m}(\boldsymbol{p}) \chi_{i}(\boldsymbol{q}) \chi_{j}(-\boldsymbol{k})^{(1)}}\right] d \boldsymbol{p} d \boldsymbol{q},(92)
\end{aligned}
$$


where the superscripts indicate the first order DIA correction, given by expressions (79) and (80). The DIA quantity expression for $T_{i j}(\boldsymbol{k})$ is obtained using the procedure outlined in section A, yielding,

$$
\begin{aligned}
T_{i j}(\boldsymbol{k}) & =k_{m} \iint \delta(\boldsymbol{k}-\boldsymbol{p}-\boldsymbol{q})\left[\Theta^{s, c}(k, p, q, t)\right. \\
& -P_{m a b}(\boldsymbol{p}) F_{a j}(\boldsymbol{k}) F_{b i}(\boldsymbol{q}) \\
& -q_{a} F_{a j}(\boldsymbol{k}) F_{m i}(\boldsymbol{p}) \\
& -q_{a} \Phi_{a m}(\boldsymbol{p}) \Psi_{i j}(\boldsymbol{k}) \\
& +k_{a} \Phi_{a m}(\boldsymbol{p}) \Psi_{j i}(\boldsymbol{q}) \\
& \left.+k_{a} F_{a i}(\boldsymbol{q}) F_{m j}(\boldsymbol{p})\right] d \boldsymbol{p} d \boldsymbol{q}
\end{aligned}
$$

which gives, for the transfer of the solenoidal and compressible part, respectively, of the displacement vector variance,

$$
\begin{aligned}
T^{s} & \equiv 2 \pi k^{2} P_{i j}(\boldsymbol{k}) T_{i j}(\boldsymbol{k}) \\
& =\frac{1}{4} \iint_{\Delta} \Theta^{s}(k, p, q, t)[ \\
& -\left(x y+z^{3}-z+z x^{2}\right) p^{3} F(k) F(q) \\
& -z(x+y z) q^{3} F(k) F(p) \\
& -2\left(1-z^{2}\right) k q^{2} \Psi^{s}(k) E(p) \\
& +\left(1-z^{2}\right)\left(1+y^{2}\right) k^{3} \Psi^{s}(q) E(p) \\
& +\left(1-z^{2}\right)\left(1-y^{2}\right) k^{3} \Psi^{c}(q) E(p) \\
& \left.+\left(-x y z-y^{2} z^{2}\right) k^{3} F(p) F(q)\right] \frac{d p}{p} \frac{d q}{q}
\end{aligned}
$$

and

$$
\begin{aligned}
T^{c} & \equiv 4 \pi k^{2} \Pi_{i j}(\boldsymbol{k}) T_{i j}(\boldsymbol{k}) \\
& =\frac{1}{2} \iint_{\Delta} \Theta^{c}(k, p, q, t)[ \\
& -\left(1-z^{2}\right) k q^{2} \Psi^{c}(k) E(p) \\
& +\left(1-z^{2}\right)\left(1-y^{2}\right) k^{3} \Psi^{s}(q) E(p) \\
& +\left(1-z^{2}\right)\left(y^{2}\right) k^{3} \Psi^{c}(q) E(p) \\
& \left.+\left(1-z^{2}\right)\left(1-y^{2}\right) k^{3} F(p) F(q)\right] \frac{d p}{p} \frac{d q}{q} .
\end{aligned}
$$

The symbol $\Delta$ indicates the domain in the $p q$-plane in which $k, p, q$ can form a triangle (in other words $|p-q| \leq k \leq|p+q|$ ) and $x, y, z$ are given by

$$
\begin{gathered}
x=-p_{i} q_{i} /(p q) \\
y=k_{i} q_{i} /(k q) \\
z=k_{i} p_{i} /(k p) .
\end{gathered}
$$




\section{A model for the displacement triple correla- tion}

The closed expressions for $\Xi^{I}(k), \Xi^{I I}(k)$ and $\Xi^{I I I}(k)$ are obtained by the same procedure outlined in the previous section, leading to

$$
\begin{aligned}
& S_{\|}^{(3)}(r)=-6 \frac{r_{i} r_{j} r_{m}}{r^{3}} \int \Xi_{i j m}(\boldsymbol{k})\left[1-e^{i \boldsymbol{k} \cdot \boldsymbol{r}}\right] d \boldsymbol{k}, \\
& =-3 i r \int \Theta^{\Xi}(k, p, q) j(k r) \\
& {\left[-\quad y(x+y z) p^{3} F(k) E^{s}(q)\right.} \\
& +y(x+y z) p^{3} F(k) E^{c}(q) \\
& \text { - } \quad(z+x y) p^{3} F(q) E^{s}(k) \\
& +\frac{1}{2}\left(1-2 x y z-3 y^{2} z^{2}+y^{2}-z^{2}\right) k^{3} F(p) E^{s}(q) \\
& \left.+\frac{1}{2}\left(1+2 x y z+3 y^{2} z^{2}-y^{2}-z^{2}\right) k^{3} F(p) E^{c}(q)\right] \\
& +\Theta^{\Xi}(k, p, q) l(k r) \\
& \text { [- }(z+x y) p^{3} F(q) E^{c}(k) \\
& +\left(1-z^{2}\right)\left(1-y^{2}\right) k^{3} F(p) E^{s}(q) \\
& \left.+\quad\left(1-z^{2}\right) y^{2} k^{3} F(p) E^{c}(q)\right] \frac{d p}{p} \frac{d q}{q} d k
\end{aligned}
$$

\section{Closure of the energy and scalar flux spec- trum}

A standard closure expression for the energy transfer in the Lin-equation is

$$
\iint_{\Delta} \Theta^{E}(k p q)\left[x y+z^{3}\right] p E(q)\left[k^{2} E(p)-p^{2} E(k)\right] \frac{d p d q}{p q} .
$$

This form is common to a number of closures such as EDQNM, DIA, Test-FieldModel, LMFA closure. The closure for the scalar flux spectrum is

$$
\left[\frac{\partial}{\partial t}+(\nu+D) k^{2}\right] F(k)=\sum_{i=1}^{8} T_{i}(k)+E(k)
$$


with

$$
\begin{aligned}
& T_{1}(k)=-\frac{1}{8} \int_{\Delta} \Theta^{F}(k p q) f_{1}(k, p, q) p^{3} E(q) F(k) \frac{d p}{p} \frac{d q}{q} \\
& T_{2}(k)=-\frac{1}{8} \int_{\Delta} \Theta^{F}(k p q) f_{2}(k, p, q) q^{3} E(p) F(k) \frac{d p}{p} \frac{d q}{q} \\
& T_{3}(k)=+\frac{1}{8} \int_{\Delta} \Theta^{F}(k p q) f_{3}(k, p, q) k^{3} E(p) F(q) \frac{d p}{p} \frac{d q}{q} \\
& T_{4}(k)=+\frac{1}{8} \int_{\Delta} \Theta^{F}(k p q) f_{4}(k, p, q) k^{3} E(q) F(p) \frac{d p}{p} \frac{d q}{q} \\
& T_{5}(k)=-\frac{1}{4} \int_{\Delta} \Theta^{F}(q p k) f_{5}(k, p, q) p^{3} E(k) F(q) \frac{d p}{p} \frac{d q}{q} \\
& T_{6}(k)=+\frac{1}{4} \int_{\Delta} \Theta^{F}(q p k) f_{6}(k, p, q) k^{3} E(p) F(q) \frac{d p}{p} \frac{d q}{q} \\
& T_{7}(k)=-\frac{1}{4} \int_{\Delta} \Theta^{F}(q p k) f_{7}(k, p, q) q^{3} E(k) F(p) \frac{d p}{p} \frac{d q}{q} \\
& T_{8}(k)=-\frac{1}{4} \int_{\Delta} \Theta^{F}(q p k) f_{8}(k, p, q) q^{3} E(p) F(k) \frac{d p}{p} \frac{d q}{q} .
\end{aligned}
$$

with

$$
\begin{aligned}
& f_{1}=(k p)^{-1} P_{123}(\boldsymbol{k}) P_{214}(\boldsymbol{p}) P_{34}(\boldsymbol{q})=2\left(x y+z^{3}\right) \\
& f_{2}=(k q)^{-1} P_{123}(\boldsymbol{k}) P_{24}(\boldsymbol{p}) P_{314}(\boldsymbol{q})=2\left(x z+y^{3}\right) \\
& f_{3}=(k)^{-2} k_{1} P_{234}(\boldsymbol{k}) P_{13}(\boldsymbol{p}) P_{24}(\boldsymbol{q})=1-x y z-2 y^{2} z^{2}+y^{2}-z^{2} \\
& f_{4}=(k)^{-2} k_{1} P_{234}(\boldsymbol{k}) P_{23}(\boldsymbol{p}) P_{14}(\boldsymbol{q})=1-x y z-2 y^{2} z^{2}-y^{2}+z^{2} \\
& f_{5}=(k p)^{-1} k_{1} P_{23}(\boldsymbol{k}) P_{124}(\boldsymbol{p}) P_{34}(\boldsymbol{q})=x y+z^{3}-z+z x^{2} \\
& f_{6}=(k)^{-2} k_{1} P_{234}(\boldsymbol{k}) P_{13}(\boldsymbol{p}) P_{24}(\boldsymbol{q})=f_{3} \\
& f_{7}=(k q)^{-1} k_{1} P_{23}(\boldsymbol{k}) P_{13}(\boldsymbol{p}) q_{2}=z(x+y z) \\
& f_{8}=2(k q)^{-1} k_{1} P_{12}(\boldsymbol{p}) q_{2}=2(y+x z) .
\end{aligned}
$$

Note that $f_{4}$ and $f_{2}$ are not needed when symmetry is used to show that $T_{1}(k)=$ $T_{2}(k)$ and $T_{3}(k)=T_{4}(k)$. Since $f_{6}=f_{3}$, we eventually only need five different factors. This form $[36,22]$ is a corrected form of the expression by [26], see also [41].

\section{References}

[1] J. C. R. Hunt and D. J. Carruthers, Rapid distortion theory and the problems of turbulence, J. Fluid Mech. 212, 497 (1990).

[2] C. Cambon and J. F. Scott, Linear and nonlinear models of anisotropic turbulence, Ann. Rev. Fluid Mech. 31, 1 (1999). 
[3] T. Ishihara, K. Yoshida, and Y. Kaneda, Anisotropic Velocity Correlation Spectrum at Small Scales in a Homogeneous Turbulent Shear Flow, Phys. Rev. Lett. 88, 154501 (2002).

[4] Y. Kaneda and K. Yoshida, Small-scale anisotropy in stably stratified turbulence, New J. Phys. 6, 34 (2004).

[5] P. Sagaut and C. Cambon, Homogeneous Turbulence Dynamics, Cambridge University Press, 2008.

[6] A. Kolmogorov, The local structure of turbulence in incompressible viscous fluid for very large Reynolds numbers, Dokl. Akad. Nauk. SSSR 30, 301 (1941).

[7] S. Corrsin, Heat transfer in isotropic turbulence, J. Appl. Phys. 23(1), 113-118 (1952).

[8] P. Mestayer, Local isotropy and anisotropy in a high-Reynolds-number turbulent boundary layer, J. Fluid Mech. 125, 47 (1982).

[9] K. R. Sreenivasan and R. A. Antonia, Skewness of temperature derivatives in turbulent shear flows, Phys. Fluids 20, 1986 (1977).

[10] A. Obukhov, Structure of the temperature field in turbulent flows, Isv. Geogr. Geophys. Ser. 13, 58 (1949).

[11] L. Mydlarski and Z. Warhaft, Passive scalar statistics in high Peclet number grid turbulence, J. Fluid Mech. 358, 135 (1998).

[12] A. Pumir, A numerical study of the mixing of a passive scalar in three dimensions in the presence of a mean gradient, Phys. Fluids 6, 2118 (1994).

[13] M. Holzer and E. Siggia, Turbulent mixing of a passive scalar, Phys. Fluids A 6, 1820 (1994).

[14] A. Pumir and B. Shraiman, Persistent Small Scale Anisotropy in Homogeneous Shear Flows, Phys. Rev. Lett. 75, 3114 (1995).

[15] S. Garg and Z. Warhaft, On the small scale structure of simple shear flow, Phys. Fluids 10, 662 (1998).

[16] X. Shen and Z. Warhaft, The anisotropy of the small scale structure in high Reynolds number $\left(R_{\lambda} \sim 1000\right)$ turbulent shear flow, Phys. Fluids 12, 2976 (2000).

[17] S. Kurien, K. Aivalis, and K. R. Sreenivasan, Anisotropy of small-scale scalar turbulence, J. Fluid Mech. 448, 279 (2001).

[18] L. Mydlarski, A. Pumir, B. Shraiman, E. Siggia, and Z. Warhaft, Structures and Multipoint Correlators for Turbulent Advection: Predictions and Experiments, Phys. Rev. Lett. 81, 4373 (1998). 
[19] L. Mydlarski and Z. Warhaft, Three-point statistics and the anisotropy of a turbulent passive scalar, Phys. Fluids 10, 2885 (1998).

[20] S. Corrsin, On the spectrum of isotropic temperature fluctuations in an isotropic turbulence, J. Appl. Phys. 22, 469 (1951).

[21] J. Lumley, Similarity and the turbulent energy spectrum, Phys. Fluids 10, 855 (1967).

[22] W. Bos and J.-P. Bertoglio, Lagrangian Markovianized Field Approximation for turbulence, J. Turbul. 14, 99 (2013).

[23] G. K. Batchelor, The theory of homogeneous turbulence, Cambridge University Press, 1953.

[24] W. Bos, L. Chevillard, J. Scott, and R. Rubinstein, Reynolds number effect on the velocity increment skewness in isotropic turbulence, Phys. Fluids 24, 015108 (2012).

[25] J. R. Herring, Approach of axisymmetric turbulence to isotropy, Phys. Fluids 17, 859 (1974).

[26] S. Herr, L. Wang, and L. Collins, EDQNM model of a passive scalar with a uniform mean gradient, Phys. Fluids 8, 1588 (1996).

[27] T. Gotoh, T. Watanabe, and Y. Suzuki, Universality and anisotropy in passive scalar fluctuations in turbulence with uniform mean gradient, J. Turbul. , N48 (2011).

[28] I. Arad, V. L'vov, and I. Procaccia, Correlation functions in isotropic and anisotropic turbulence: The role of the symmetry group, Phys. Rev. E 59, 6753 (1999).

[29] R. Kraichnan, Lagrangian-History Closure Approximation for Turbulence, Phys. Fluids 8, 575 (1965).

[30] Y. Kaneda, Renormalized expansions in the theory of turbulence with the use of the Lagrangian position function, J. Fluid. Mech. 107, 131 - 145 (1981).

[31] W. Bos and J.-P. Bertoglio, A single-time two-point closure based on fluid particle displacements, Phys. Fluids 18, 031706 (2006).

[32] R. Kraichnan, An almost-Markovian Galilean-invariant turbulence model, J. Fluid Mech. 47, 513 (1971).

[33] R. Kraichnan, Kolmogorov's Hypotheses and Eulerian Turbulence Theory, Phys. Fluids 7, 1723 (1964).

[34] R. Kraichnan, Lagrangian-History Statistical theory for Burgers' equation, Phys. Fluids 11, 265 (1968). 
[35] W. Bos, R. Rubinstein, and L. Fang, Reduction of mean-square advection in turbulent passive scalar mixing, Phys. Fluids 24, 075104 (2012).

[36] W. Bos, H. Touil, and J.-P. Bertoglio, Reynolds number dependency of the scalar flux spectrum in isotropic turbulence with a uniform scalar gradient, Phys. Fluids 17, 125108 (2005).

[37] P. A. O'Gorman and D. I. Pullin, Effect of Schmidt number on the velocityscalar cospectrum in isotropic turbulence with a mean scalar gradient, J. Fluid Mech. 532, 111 (2005).

[38] T. Watanabe and T. Gotoh, Scalar flux spectrum in isotropic steady turbulence with a uniform mean gradient, Phys. Fluids 19, 121701 (2007).

[39] R. Kraichnan, The structure of isotropic turbulence at very high Reynolds numbers, J. Fluid Mech. 5, 497-543 (1959).

[40] P. Roberts, Analytical theory of turbulent diffusion., J. Fluid Mech. 11, 257 (1961).

[41] M. Ulitsky and L. Collins, On constructing realizable, conservative mixed scalar equations using the eddy-damped quasi-normal Markovian theory, J. Fluid Mech. 412, 303-329 (2000). 